\title{
Une édition des archives de Charles Duvelle (1961-1974): la collection Prophet
}

10 vol., Kora Sons/Philips, 1999

\section{François Borel}

\section{(2) OpenEdition}

\section{Journals}

Édition électronique

URL : http://journals.openedition.org/ethnomusicologie/734

ISSN : 2235-7688

Éditeur

ADEM - Ateliers d'ethnomusicologie

Édition imprimée

Date de publication : 1 janvier 2001

Pagination : 243-247

ISBN : 2-8257-0723-6

ISSN : $1662-372 X$

Référence électronique

François Borel, «Une édition des archives de Charles Duvelle (1961-1974): la collection Prophet », Cahiers d'ethnomusicologie [En ligne], 13|2001, mis en ligne le 09 janvier 2012, consulté le 25 avri 2019. URL : http://journals.openedition.org/ethnomusicologie/734

Ce document a été généré automatiquement le 25 avril 2019

Tous droits réservés 


\section{Une édition des archives de Charles Duvelle (1961-1974): la collection Prophet}

10 vol., Kora Sons/Philips, 1999

\section{François Borel}

\section{RÉFÉRENCE}

Une édition des archives de Charles Duvelle (1961-1974): la collection Prophet. Prophet 01: Tchad; 02: Sénégal; 03: Papua New Guinea; 04: Niger; 05: Mauritanie; 06: Madagascar; 07: Congo; 08: Centrafrique; 09: Burkina Faso; 10: Bénin. Enregistrements, textes et photos réalisés par Charles Duvelle. 10 CDs Kora Sons/Philips, 1999. (Remastérisation en haute définition réalisée à partir des masters originaux entièrement digitalisés et équalisés en 20 bits).

1 Une première série de dix disques $\mathrm{CD}$ (déjà suivie d'une deuxième série parue en 2000): voilà ce qu'attendaient tous les habitués des albums vinyl 45 et $33 \mathrm{t}$. SOR (Sorafom) et OCR des années soixante associant le nom de Charles Duvelle à celui du fameux Office de co opération Radiophonique, institution destinée à promouvoir les cultures musicales traditionnelles du monde entier, et en particulier celles des anciennes colonies et territoires français d'Outre-Mer. Cette nouvelle collection, éditée sous la marque Kora Sons (!), affiliée à Philips, réunit des documents presque tous inédits, recueillis en Afrique, à Madagascar et en Papouasie Nouvelle-Guinée de 1961 à 1974.

2 Chacun des dix volumes de cette première série Prophet se présente sous la forme d'un élégant livret cartonné habillé d'une photographie pleine page en couleurs, accompagné d'une brochure de 20 ou 24 pages offrant à l'auditeur, en français et en anglais, des informations, ainsi qu'une carte pour la localisation géographique des enregistrements. Ceux-ci, toujours de bonne qualité, font l'objet d'une description parfois succincte, parfois 
plus abondante, selon la documentation à disposition, l'auteur s'étant aussi adjoint la collaboration occasionnelle d'un membre du groupe étudié. D'excellentes photographies en noir et blanc et parfois en couleurs illustrent le propos. Un texte d'introduction, commun à tous les $\mathrm{CD}$, énumère les nombreux atouts de cette collection: qualité sonore, originalité et ancienneté, caractère spontané des productions musicales, respect de la durée originale des prestations, etc., ainsi qu'une biographie de Charles Duvelle.

3 Le premier volume, consacré au Tchad, livre des documents inédits complétant ceux déjà publiés dans le coffret OCR 36, 37, 38, enregistrés en 1966 par Charles Duvelle et Michel Vuylsteke et publiés en 1968. Il s'agit de musiques pour la période des récoltes, pour les rituels de guérison, les veillées de classes d'âge, les rites de passage (mariage) et les louanges, toutes recueillies dans la savane du Mayo-Kebbi, au sud-ouest du Tchad, chez les Toupouri, les Baïnaoua, les Banana, les Kado et les Moundang. Elles offrent une large panoplie instrumentale incluant aussi bien des trompes et porte-voix en calebasse, des flûtes droites et des hochets et sonnailles de cheville, que des tambours à une ou deux membranes et des xylophones portatifs. Les chants, solistes ou responsoriaux, ainsi que la danse, sont omniprésents. A noter la plage 2, «Indépendance», chantée par un joueur de vièle monocorde.

4 Avec le deuxième $\mathrm{CD}$, on passe à l'extrême ouest de l'Afrique occidentale, d'abord chez les Diola de Casamance (Sénégal) où ont été enregistrées en 1967 les diverses étapes de la cérémonie itinérante Lébounaye qui se déroule après la récolte du riz (plages 1 à 5). D’une durée de trois jours, cette fête a lieu successivement dans plusieurs villages et quartiers de villages, ceci au cours d'une période de deux mois (avril-mai) précédant l'hivernage. Aux chants de fertilité des femmes succèdent, sur un tempo très vif, des séquences instrumentales combinant des rythmes de tambours et de battements de mains, alternant avec des chœurs masculins a cappella. Un peu plus à l'est à l'intérieur du pays, près de la frontière guinéenne, les Bassari célèbrent les rites d'initiation koré, durant lesquels l'adolescent passe à l'état d'adulte sous le contrôle sévère des initiés portant les masques lukuta et dansant au son de sifflets, de sonnailles et de coups de fusil, ou tambourinant sur des tambours à une peau chevillée (plages 6-7). Suivent deux types de flûtes, traversière pour le divertissement, droite pour la marche (8-9).

5 Le troisième volume nous conduit presque aux antipodes, c'est-à-dire en Papouasie Nouvelle-Guinée (Papua New Guinea), chez les Huli du district des Southern Highlands en 1974. La musique de cette population, qui n'avait jusque-là jamais été enregistrée, offre quelques caractéristiques intéressantes: par exemple la flûte de Pan, instrument de divertissement à sept tuyaux dont le musicien tire des sons presque complètement couverts par son propre souffle (plage 2); ou ce chant de séduction dans lequel une voix déformée alterne avec le son aigrelet d'un tuyau de flûte (plage 4); ou encore ces chants de jeunes garçons en cours d'initiation qui répondent d'une voix aiguë aux injonctions de leurs initiateurs. La guimbarde à traction est présente, jouée par les femmes, leur permettant de formuler des souhaits qui ne seront compris que par les autres femmes (plage 8). Mais l'instrument le plus intéressant et le plus rarement enregistré est sans doute l'arc musical à bouche à deux cordes, qui sert à communiquer entre initiés. Ce volume mérite une mention particulière étant donné la relative rareté des publications sonores dans le domaine de la musique de Papouasie.

De retour en Afrique de l'Ouest avec les chants de louanges songhay, zarma et haoussa du Niger (volume 4, 1961), l'auditeur fait connaissance avec le style vocal des griots sahéliens. Le premier document est une musique de mariage chantée par des jeunes filles 
accompagnées d'un orchestre de tambours d'aisselle et d'un gros tambour à deux membranes. Le dernier (plage 6) présente un chanteur-tambourinaire haoussa accompagnant son fils, joueur de hautbois alghaita, se produisant pour chanter les louanges du chef zarma Djermakoye de Dosso. Entre ces deux extrêmes, les plages 2 à 5 offrent successivement un chant a cappella de N'Garin, cultivateur songhay, qui récite les dynasties de cultivateurs et les louanges à de généreux donateurs (déjà édité dans le 45 tours SOR 4); une chanson de Zeinabou, femme zarma aveugle accompagnée d'un joueur de vièle monocorde godié (voir SOR 5); puis un chant accompagné au luth monocorde kontigui, par Boubou Sanda, chanteur-instrumentiste, suivi d'une pièce chantée par un adolescent, Doulo Soumahilou, accompagné par Ibrahima Douma et par les battements de mains de six jeunes gens. Ce dernier chant (voir SOR 4) est remarquable par la virtuosité de l'instrumentiste qui s'adapte subtilement au timbre et aux nuances de la voix du jeune chanteur, soutenu rythmiquement par les interventions parfaitement maitrisées des battements de mains. Il est en outre heureux que la traduction des textes des chants figure, même partiellement, dans le livret.

7 Du Niger, on passe en Mauritanie orientale, plus précisément dans le Hodh, dont Charles Duvelle a fréquenté des griots et griottes maures en 1965. Dans la notice de ce volume 5 ( Mauritanie - concert sous la tente), il décrit tout d'abord le système des trois «voies» de la musique maure (noire, blanche, tachetée). Celles-ci sont basées sur une succession de modes dont le chanteur et joueur de luth à trois cordes tidinit se doit de respecter l'ordre, au besoin en annonçant à son public le titre de la pièce qu'il va jouer tout en accordant son instrument. Le rôle des femmes griottes est ici d'accompagner le chanteur soit en se contentant de frapper à certains moments la table d'harmonie de leur instrument (plage 1), la harpe ardin, soit en jouant et chantant également (plages 2-4). L'intérêt de ce disque réside surtout dans la reproduction presque intégrale d'un «concert sous la tente» (plage 1, 33'54), dans les arrêts de jeu et les remarques des musiciens, fidèlement conservés, qui confèrent au document toute son authenticité. Les explications de l'auteur sont claires, complétées par un schéma indispensable à la compréhension de la structure complexe de cette musique.

8 L'auteur a sillonné l'île de Madagascar du nord au sud en 1963. Il en a rapporté une copieuse moisson d'enregistrements couvrant la majorité des groupes ethniques et des instruments de musique utilisés dans de nombreux contextes. Ce volume 6 est évidemment en grande partie consacré au répertoire joué à la cithare valiha (prononcer «vali») sous ses différentes formes: à 18 cordes métalliques et à caisse parallélépipédique en bois sur les deux premières plages (musique betsimisaraka); à 14, 16 ou 18 cordes sur une caisse tubulaire en bambou, forme la plus répandue, dans les plages 3 et 4 (musique sakalava), où elle accompagne des épopées chantées. Mais un autre cordophone est caractéristique de l'île de Madagascar, le violon, que l'on peut entendre sous sa forme occidentale ou locale, accompagnant un chant funèbre antanosy (plage 5), une fête de circoncision bara (plage 8) et un spectacle merina (plage 10). Deux instruments sont encore à mentionner: la cithare sur bâton à neuf cordes métalliques lokanga, pourvue d'un résonateur en calebasse appuyé contre la poitrine pour varier le timbre de l'instrument (plage 9) et un bidon enveloppant la tête d'un mendiant aveugle qui en frappe les parois tout en chantant une mélopée inspirée d'un chant indien.

9 Le volume 7 (1966) est dévolu au sud du Congo-Brazzaville. Il s'ouvre sur une cérémonie de lever de deuil balari avec danse mixte, chant masculin responsorial accompagné de tambours ngoma et de hochets en boîte de conserve. Suit une complainte balari chantée 
par un joueur de pluriarc, puis une danse où s'exprime une chanteuse sur le thème de la femme sans enfants. Une cérémonie de deuil d'un notable bakongo permet d'entendre sept trompes en ivoire jouées avec la technique du hoquet (chaque instrument joue une seule note alternativement) et accompagnées de timbales (plage 4). Le document le plus intéressant est certainement celui qui fait entendre les trompes anthropomorphes bembé, instruments déjà tombés en désuétude à l'époque où Charles Duvelle les a enregistrés. C'est pourquoi les renseignements au sujet de la fonction de ces instruments font défaut, comme l'auteur ne peut que le constater.

10 La musique des Pygmées Babinga de Centrafrique (1962) constitue la moitié des documents réunis dans le disque 8. Les instruments utilisés ici vont du xylophone à la harpe-cithare, en passant par la sanza, les tambours et les sifflets, notamment sur la plage 2 , où ces derniers alternent rythmiquement avec la voix des joueurs. Ce magnifique chant de chasse est suivi d'un autre combinant le son aigrelet de la harpe-cithare à un accompagnement de battements de mains et de voix de tête. Leur succède une danse rythmée de manière très complexe par des tambours, des battements de mains et des voix d'hommes. Divers cris d'animaux imités par un chasseur illustrent la fonction principale de toutes ces musiques: la chasse. La musique des Bofi et des Isongo, voisins des Babinga, est représentée notamment par une musique de divinitation, le jeu de la harpe arquée à dix cordes ngombi, ainsi que du chant ioulé (technique du yodel).

11 Les enregistrements réalisés au sud du Burkina Faso (alors Haute-Volta) en 1961 constituent le neuvième volume. Duvelle en consacre deux plages importantes aux xylophones des Lobi, dont le premier est joué lors de funérailles (plage 1), alors que les deux autres sont entendus dans le cadre d'une danse, joués en une combinaison démontrant la virtuosité des musiciens (plage 2) et des tambourinaires, tandis que les danseurs, munis de sonnailles métalliques au genou, ajoutent encore un élément syncopé à l'ensemble. Une autre plage (5) fait entendre des danseurs bisa en couples mixtes portant des sonnailles de chevilles qui constituent le seul élément rythmique soutenant le chant. L'arc en bouche est représenté par le chant d'amour d'un musicien gan qui s'accompagne lui-même de son instrument. Le disque se termine sur un chant «licencieux» mossi accompagné au luth koundé (plage 7) dont on aurait bien voulu connaitre les paroles afin de partager les rires de l'assistance.

Le dernier volume de la série est entièrement consacré à la musique des Bariba enregistrée dans la région de Parakou, au Bénin (alors Dahomey) en 1962. La nature et le jeu des instruments évoquent irrésistiblement la musique des puissants voisins des Bariba, les Haoussa du Nigéria. C'est le cas des premières plages, notamment d'une danse d'intronisation des rois bariba où la base rythmique est assurée par un gros tambour accompagné d'un tambour d'aisselle à tension variable; ou de ces deux vièles monocordes qui alternent pour accompagner le chant de louanges à une divinité de la fécondité, soutenues par le rythme d'une calebasse frappée et d'un gros tambour gon. Ce dernier se fait aussi entendre lors de veillées pendant la période du Ramadan, pour accompagner les chants de louanges aux cultivateurs. Ici, on distingue nettement la vibration due au timbre (ficelle tendue effleurant la peau du tambour). Plus loin (plage 7), une femme chante en solo, s'accompagnant d'une cithare en radeau qu'elle utilise surtout comme instrument de percussion. Présent aussi dans cette population, le xylophone sur cuisses, fait de quatre bûches percutées à l'aide de deux pierres, est utilisé pour éloigner des champs cultivés les singes et les oiseaux. 
13 La qualité de cette première série est incontestable. Le choix des enregistrements est toujours intelligent: peu de récurrences, une judicieuse répartition des instruments, une durée appropriée, la seule réserve dans ce domaine étant l'utilisation trop systématique du fade out. En effet, certaines plages auraient gagné à être allongées, d'autant plus que la durée de chaque volume se situe en moyenne autour de 55 minutes. Quant aux commentaires des enregistrements, ils ont manifestement été partiellement repris des anciens albums SOR et OCR. Si la teminologie vernaculaire et le nom des musiciens y figurent presque toujours, ils sont parfois un peu brefs et se limitent trop souvent aux seules descriptions organologiques. Certains textes de chants sont livrés en traduction, mais jamais en transcription vernaculaire. Ces quelques lacunes sont compensées par la qualité des photographies qui permettent de compléter l'information. Cette collection Prophet se doit de figurer dans toutes les discothèques. 